\title{
Correction to: Nationwide study of paroxysmal nocturnal hemoglobinuria in South Korea: paradox of eculizumab
}

\author{
Ka-Won Kang ${ }^{1} \cdot$ Hyemi Moon ${ }^{2}$ Byung-Hyun Lee ${ }^{1} \cdot$ Min Ji Jeon ${ }^{1}$ - Eun Sang Yu ${ }^{1}$. Dae Sik Kim ${ }^{1} \cdot$ Se Ryeon Lee ${ }^{1}$. \\ Hwa Jung Sung ${ }^{1} \cdot$ Chul Won Choi $^{1} \cdot$ Byung Soo Kim ${ }^{1}$. Juneyoung Lee ${ }^{2} \cdot$ Yong Park ${ }^{1}$
}

Published online: 7 October 2021

๑) Springer-Verlag GmbH Germany, part of Springer Nature 2021

\section{Correction to: Annals of Hematology \\ https://doi.org/10.1007/s00277-020-04133-z}

The original version of this article inadvertently contained a typographical error in the Abstract section. In the Abstract, the $p$-value demonstrating the statistically significant difference between the transfusion-independence rate for the eculizumab-treated group and the eculizumab-untreated group should be $p=0.0078$, and not $p=0.078$. The correct $p$-value ( $p=0.0078)$ has been mentioned in the main text of the manuscript, and the error is only in the Abstract section.

The original article has been correction.

Publisher's note Springer Nature remains neutral with regard to jurisdictional claims in published maps and institutional affiliations.

The original article can be found online at https://doi.org/10.1007/ s00277-020-04133-z.

Yong Park

paark76@hanmail.net

1 Division of Hematology-Oncology, Department of Internal

Medicine, Korea University College of Medicine, 73,

Goryeodae-ro, Seongbuk-gu, Seoul 02841, South Korea

2 Department of Biostatistics, Korea University College of Medicine, Seoul, South Korea 